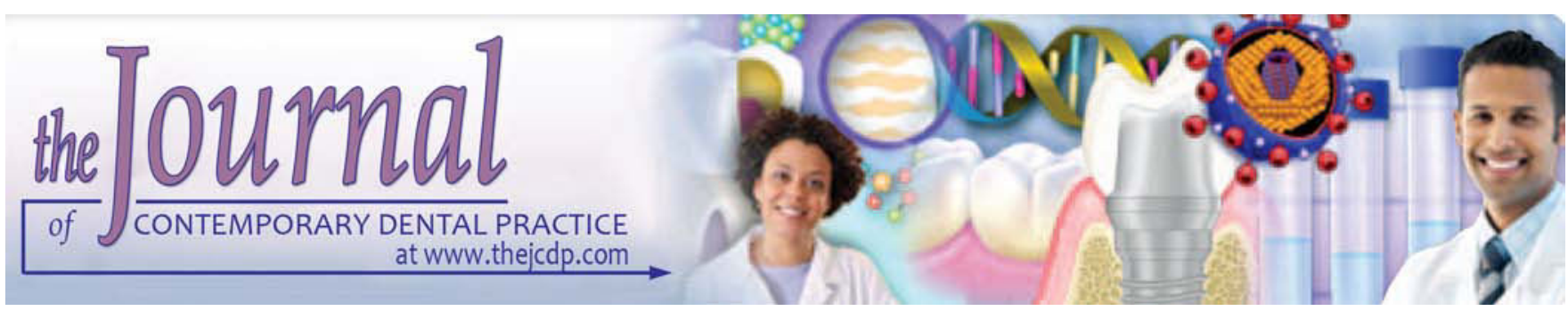

\title{
An Intraoperative Site-specific Bone Density Device: A Pilot Test Case
}

\author{
${ }^{1}$ Paolo Arosio, ${ }^{2}$ Monica Moschioni, ${ }^{3}$ Luca Maria Banfi, ${ }^{4}$ Danilo Alessio Di Stefano
}

\begin{abstract}
Aim: This paper reports a case of all-on-four rehabilitation where bone density at implant sites was assessed both through preoperative computed tomographic (CT) scans and using a micromotor working as an intraoperative bone density measurement device.
\end{abstract}

Background: Implant-supported rehabilitation is a predictable treatment option for tooth replacement whose success depends on the clinician's experience, the implant characteristics and location and patient-related factors. Among the latter, bone density is a determinant for the achievement of primary implant stability and, eventually, for implant success. The ability to measure bone density at the placement site before implant insertion could be important in the clinical setting.

Case description: A patient complaining of masticatory impairment was presented with a plan calling for extraction of all her compromised teeth, followed by implant rehabilitation. A week before surgery, she underwent CT examination, and the bone density on the CT scans was measured. When the implant osteotomies were created, the bone density was again measured with a micromotor endowed with an instantaneous torque-measuring system. The implant placement protocols were adapted for each implant, according to the intraoperative measurements, and the patient was rehabilitated following an all-on-four immediate loading protocol.

\footnotetext{
${ }^{1}$ Department of Tecnologie Biomediche Applicate Alle Scienze Odontostomatologiche, Seconda Università degli Studi di Napoli, Province: Monza e Brianza (MB), Italy

${ }^{2}$ Department of Facoltà di Medicina e Chirurgia, Università degli Studi di Brescia, Province: Brescia (BS), Italy

${ }^{3}$ Department of Facoltà di Medicina e Chirurgia, Università degli Studi di Milano, Province: Monza (MB), Italy

${ }^{4}$ Department of Dentistry, Vita e Salute San Raffaele University Milan, Italy
}

Corresponding Author: Paolo Arosio, Private Practitioner, Via Don Lualdi 4c, 20871 Ruginello, Vimercate, MB, Italy, Phone: +39039 6085229, e-mail: p.arosio@libero.it
Conclusion: The bone density device provided valuable information beyond that obtained from CT scans, allowing for site-specific, intraoperative assessment of bone density immediately before implant placement and an estimation of primary stability just after implant insertion.

Clinical significance: Measuring jaw-bone density could help clinicians to select implant-placement protocols and loading strategies based on site-specific bone features.

Keywords: Bone density, CT, СBCT, Case report, Intraoperative bone density assessment.

How to cite this article: Arosio P, Moschioni M, Banfi LM, Di Stefano DA. An Intraoperative Site-specific Bone Density Device: A Pilot Test Case. J Contemp Dent Pract 2015;16(8): 697-703.

\section{Source of support: Nil}

Conflict of interest: 'Danilo Alessio Di Stefano and Paolo Arosio have a scientific consultancy relationship with IDI Evolution.'

\section{INTRODUCTION}

The success of dental implants relies on successful osseointegration that, in turn, correlates with primary stability at the time of implant placement. ${ }^{1,2}$ Primary stability depends on several factors. ${ }^{3}$ The implant design (implant and thread shape) and surface topography are the most important implant-related ones. ${ }^{4}$ Bone density and the topographical relationship between cortical and cancellous bone at the insertion site are regarded as the most important site-related factors. ${ }^{5-9}$ Measuring bone density and assessing cortical-cancellous bone distribution at the placement site before implant insertion is, therefore, of paramount importance.

At present, bone density measurement at implant sites relies either on presurgical radiographic evaluation or intraoperatory empirical assessments. Radiographic evaluation through computed tomography (CT) or cone-beam computed tomography (CBCT) scans has been shown to provide substantial data about the topographical relation of cortical and cancellous bone at the placement site. ${ }^{10-14}$ 
Computed tomography technology may supply reliable information about bone density at the insertion site but only as an averaged value, and concerns remain about the radiation-exposure risk. ${ }^{12,14}$ A CBCT scan exposes the patient to a smaller radiation dose, but the information it gives about bone density is not absolute and may depend on the particular device used. ${ }^{15-18}$

In their daily routine, surgeons therefore, still rely on the empirical bone density classification system proposed by Misch and later modified by Trisi and Rao. ${ }^{19,20}$ In the Misch system, bone density is classified subjectively as the operator creates the osteotomy, with bone densities ranging from D1 (the hardest) to D4 (the softest). Trisi and Rao defined three classes only. Both classification systems suffer from being only qualitative, subjective, and therefore not reproducible.

Recently, a surgical micromotor has been introduced featuring an instantaneous torque-measurement system. This device measures bone density at an intermediate step of osteotomy creation, using the same principle highlighted in studies in vitro on pig ribs and jaw autopsy specimens that showed the cutting resistance to be a good estimator of bone density at the placement site. ${ }^{21,22}$ The rationale is that resistance at cutting or at probing correlates significantly with bone density, because of the greater friction exerted by denser bone. A study on bovine ribs has shown that the device provides reliable bone density values, significantly correlating with actual histomorphometric data. ${ }^{23}$ When used on patients, the device enabled zones of the jaws with different bone densities to be distinguished. ${ }^{24}$

Additionally, the device allows instantaneous insertion torque and other correlated values to be recorded at the time of implant placement. It has also been shown that the torque-depth function integral the device provides at implant insertion, correlates with immediate boneto-implant contact (BIC) at insertion in experiments on bovine ribs, later confirmed by observations on human subjects. ${ }^{23,25}$ The device, therefore, seems to convey pivotal information to the surgeon; both, immediately before implant placement, helping to design a proper implantinsertion intraoperative strategy, and just after insertion, allowing assessment of the primary stability achieved.

In this study, a case is presented in which bone density data were acquired using both conventional CT scans and the torque-measuring system, in order to get an inthe-field assessment of the advantages provided by the device, if any.

\section{CASE REPORT}

The patient was a 49-year-old woman whose masticatory function was impaired because of teeth 33 to 45 being compromised (Figs 1A and B). She was searching for functional restoration. A rehabilitation plan was designed calling for extraction of her remaining eight mandibular teeth, followed by immediate placement of two tilted implants in positions 45 and 35, two nontilted implants in positions 42 and 32, and rehabilitation using an 'allon-four' approach. The patient's informed consent was obtained.

The CT scans were analyzed using dedicated software (3Diagnosis, 3DIEMME, Italy) in order to assess the bone density at the planned insertion sites (Figs 2A to D). For each site, radiodensity (in Hounsfield Units, HU) was measured along a plane as close as possible to the surgical one. Positions of the insertion planes on the digital files were selected in relation to the most evident anatomic references, such as the socket limits or, for element 35 , the emergence of the alveolar nerve. The CT scans were also analyzed to assess the amount and spatial relationship of cortical and cancellous bone at each planned placement site.

Antibiotic prophylaxis (Amoxicillin/Clavulanic acid, Augmentin, GlaxoSmithKline, Verona, Italy) 1 gm-1 hour before surgery and then every 12 hours for 3 days, analgesic therapy (Ibuprofen, Brufen, Abbott, Roma, Italy) 400 mg three times a day for 3 days, and anti-inflammatory therapy (Dexamethasone, Decadron, Visufarma, Roma, Italy) $4 \mathrm{mg}$ just before surgery were initiated, and the patient was subjected to mouth rinses with chlorhexidine $0.2 \%$ (Corsodyl, GlaxoSmithKline, Verona, Italy), to be continued twice a day for 2 weeks after surgery. The surgical area was anesthetized with articain hydrochloride $40 \mathrm{mg} / \mathrm{ml}$ with adrenaline 1:100,000.

A single experienced surgeon (PA) did the surgery as follows: a $1 \mathrm{~cm}$ incision was performed in a palatal position of the alveolar crest, and a full-thickness flap was partially elevated to expose the underlying bone ridge. Subsequently, all elements of the lower arch were extracted atraumatically. To create the osteotomies, the surgeon first created a $2.2 \mathrm{~mm}$ round hole through the cortical bone layer and subsequently used a $2.3 \mathrm{~mm}$ bur to the depth of $8 \mathrm{~mm}$ (the desired implant placement depth). Before enlarging this to its final diameter, a bone reamer was used to drill a $3 \mathrm{~mm}$ deep, $3 \mathrm{~mm}$ wide access hole that eliminated the initial overly dense cortical bone layer and allow proper bone density measurement. The device used both to measure the bone density and later to prepare the implant sites was a TMM2 surgical micromotor (IDI Evolution, Concorezzo, Italy). During the bone density measurement, a dedicated probe was mounted on the handpiece (Fig. 3A). This probe is a $2 \mathrm{~mm}$ wide cylinder featuring equally spaced threads; its width is $3 \mathrm{~mm}$ and its shape is a $1^{\circ}$ reverse cone. After switching 

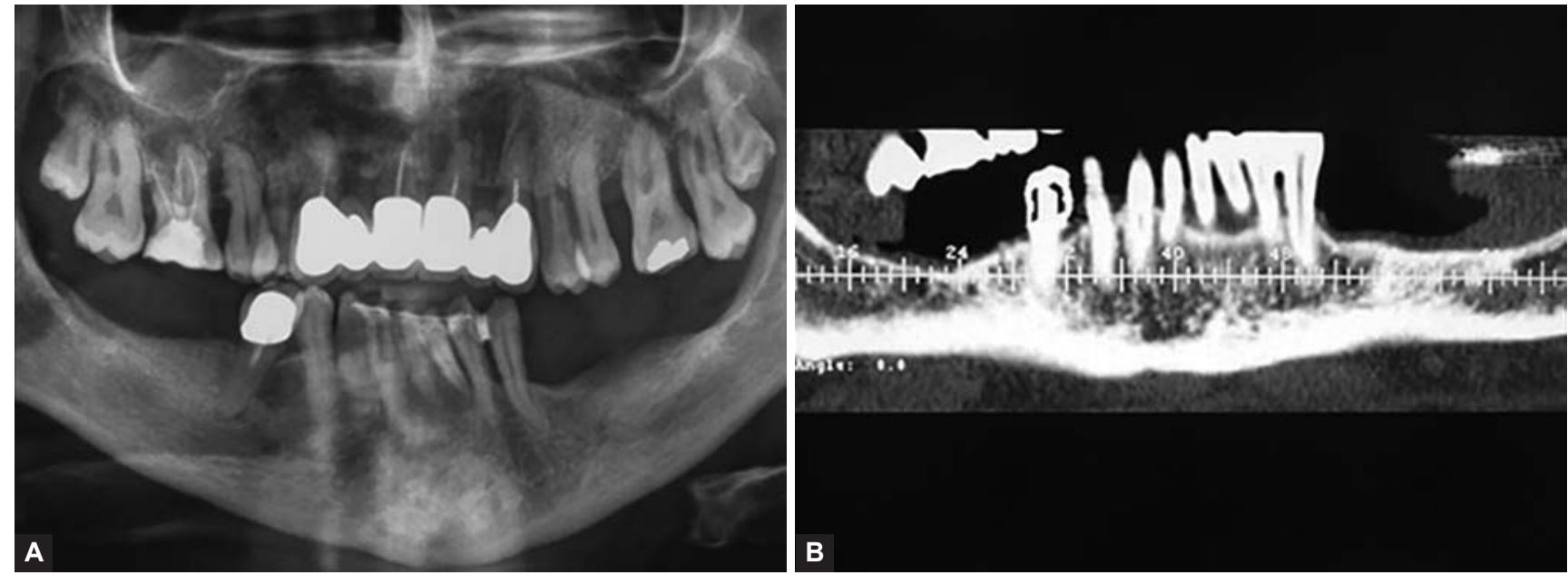

Figs $1 A$ and $B$ : Preoperative radiograph $(A)$ and $C T$ scan $(B)$
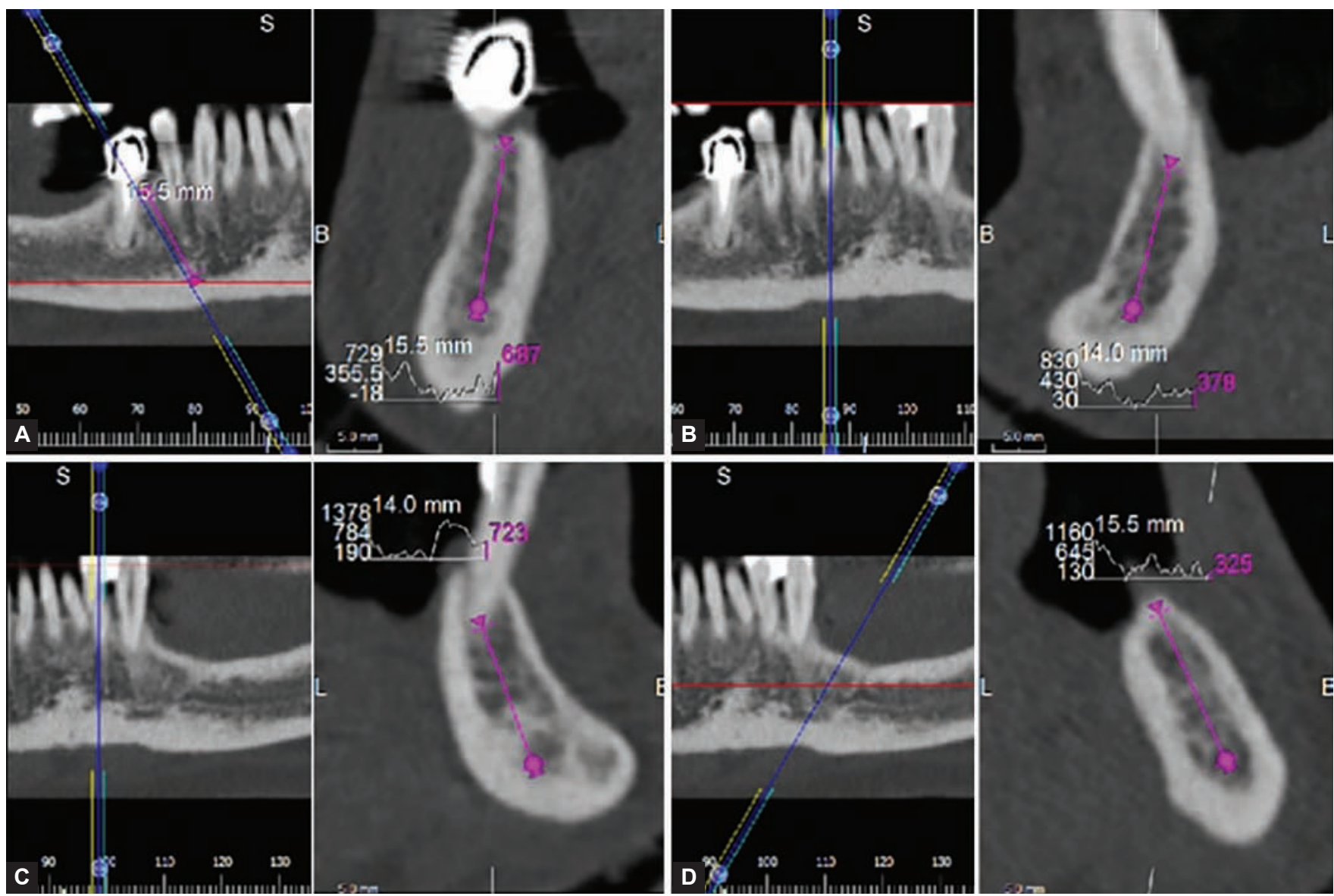

Figs $2 \mathrm{~A}$ to $\mathrm{D}$ : For each site, a panoramic projection is presented. The planned insertion axis and the corresponding section in position $45(\mathrm{~A}), 42(\mathrm{~B}), 32(\mathrm{C})$ and $35(\mathrm{D})$ are shown

the handpiece to its measurement mode, the first probe thread was inserted into the access hole. The surgeon proceeded to switch on rotation and allow the probe to screw into the previously prepared tunnel, without exerting any additional pressure (Fig. 3B). The upsidedown cone shape of the threads allowed the device to measure the friction encountered by the first thread only. When the device was in its measurement mode, the probe rotated at a given speed $(30 \mathrm{rpm})$ and could reach $35 \mathrm{~N} \times \mathrm{cm}$ maximum torque. While the probe deepened into the tunnel, software performed a high frequency sample measurement of the instantaneous torque needed to keep the speed constant. Given that the probe threads were evenly spaced and their pitch was known, the device also recorded the depth the probe had reached. This allowed a torque-depth graph to be generated which displayed how the instantaneous torque varied according to the probe depth (Figs $4 \mathrm{~A}$ to D). In addition, for each measurement, the device showed the torque peak $(\mathrm{Cp})$ and average $(\mathrm{Cm})$, the value of the torque-depth curve 
integral (I), and the maximum depth reached by the probe $(\mathrm{P})$. Instantaneous torque may be regarded as a pointto-point estimator of bone density along the tunnel, the rationale being that the denser the bone, the greater the friction on the probe thread, as well as the torque needed to keep the rotation speed constant. In turn, the average torque at probing may be regarded as an estimate of the average bone density along the whole tunnel. Data collection was supervised by a single operator trained in the detection method and recorded in the device's solidstate memory to be downloaded later for further analysis.

Cylindrical implants (Aries, IDI Evolution, Concorezzo, Italy), featuring a body diameter of $3.40 \mathrm{~mm}$, a thread diameter of $3.75 \mathrm{~mm}$, and a smooth $4.00 \mathrm{~mm}$ diameter head that allowed for supracrestal implant placement, were chosen for all sites. The implant length was $14.0 \mathrm{~mm}$ for sites 42 and 32, and $15.5 \mathrm{~mm}$ for sites 45 and 35 . Sites were prepared in accordance with the bone density measurements and the torque-depth plot obtained with the micromotor (Figs $4 \mathrm{~A}$ to D): site 45, with the lowest density values, was under-prepared at $3 \mathrm{~mm}$. Sites 42 and 35 were under-prepared at $3.2 \mathrm{~mm}$, while site 32 was prepared at $3.4 \mathrm{~mm}$. While placing each implant, the device recorded the instantaneous torque and displayed this as a torque-depth plot, along with the calculated average torque, peak torque, and the integral of the torque-depth curve at insertion (Figs 5A to D).

Bone density measurements from the preoperative CT scans and the correspondence between CT HU units and Misch classification classes are depicted in Tables 1 and 2,
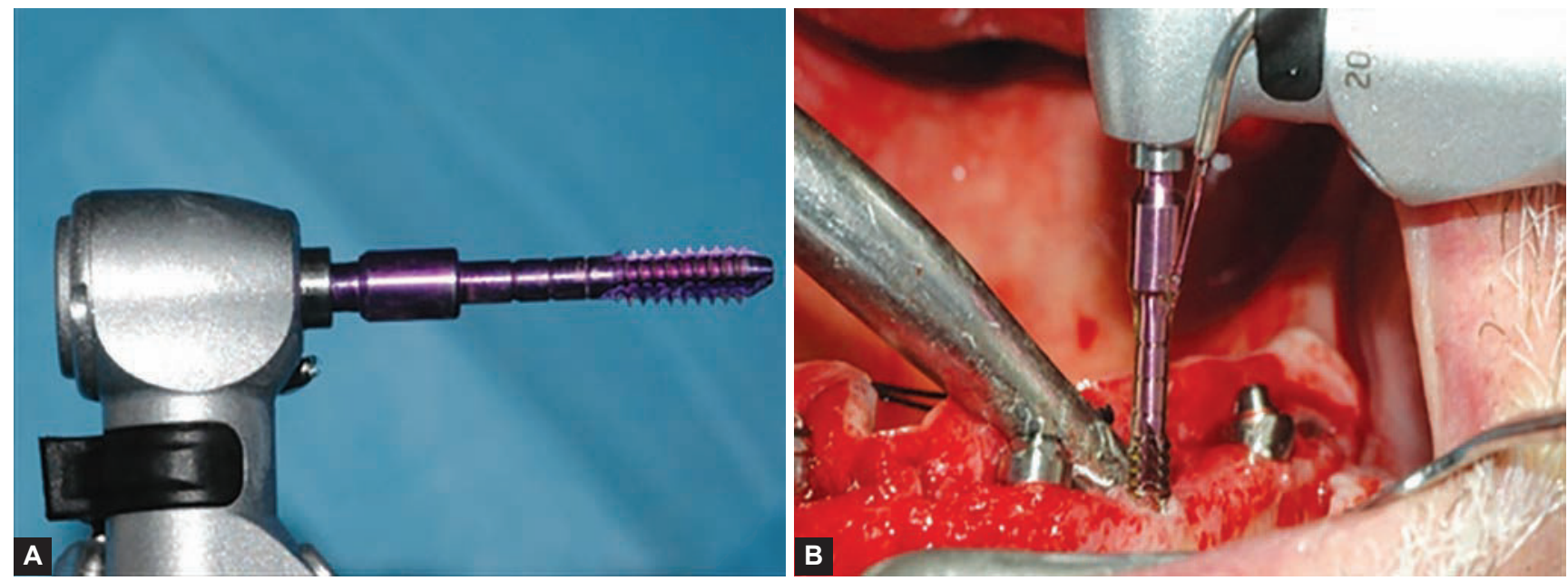

Figs $3 A$ and $B$ : The device probe $(A)$ and the measurement $(B)$
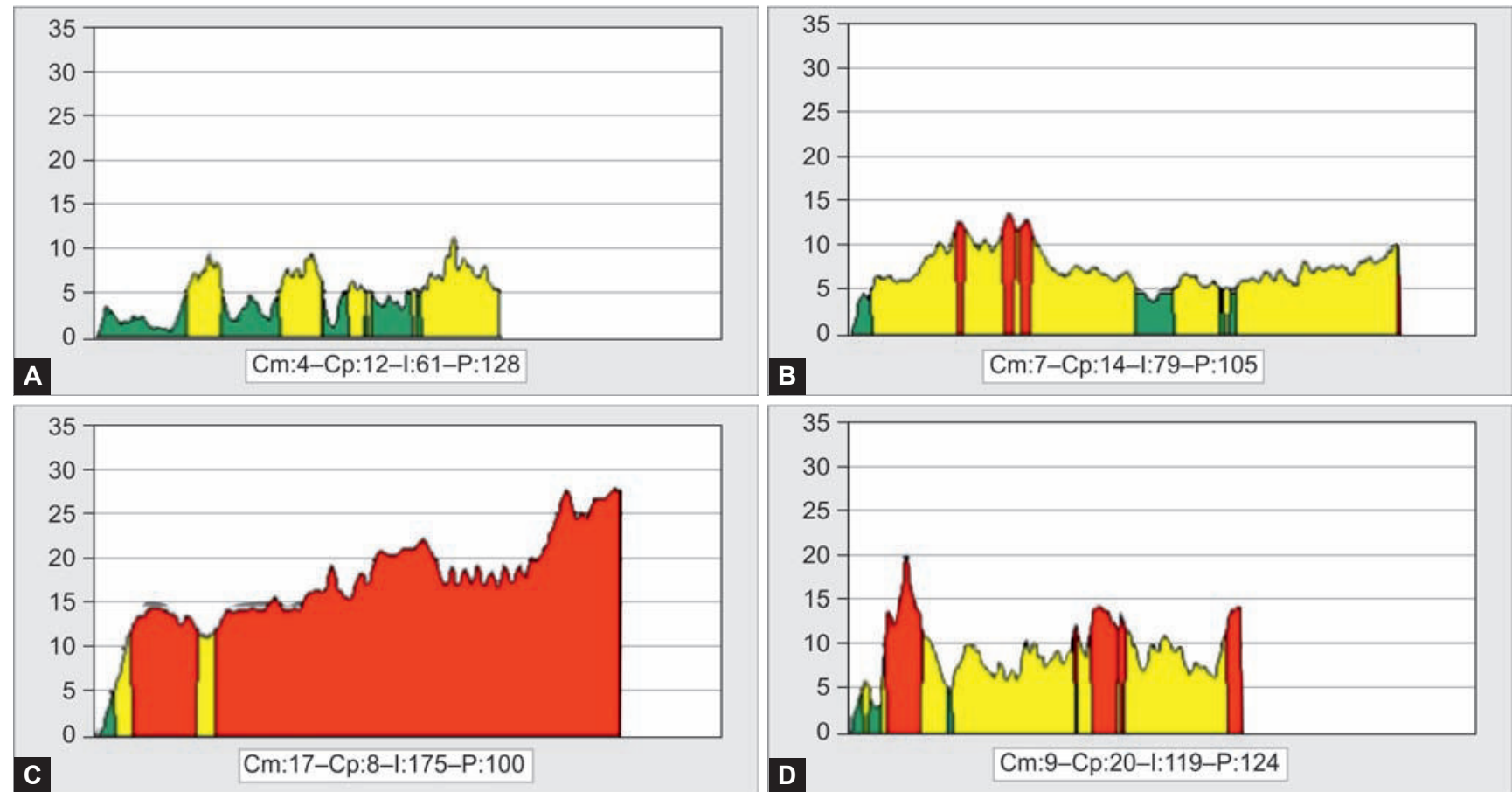

Figs 4A to $D$ : The instantaneous torque plots recorded during the intraoperative density assessment at each implant site position: 45 (A), 42 (B), 32 (C) and 35 (D). Cm: Average torque, $\mathrm{Cp}$ : Peak torque, I: Integral of the torque-depth curve, P: Maximum depth reached by the probe (in tenths of millimeters) 

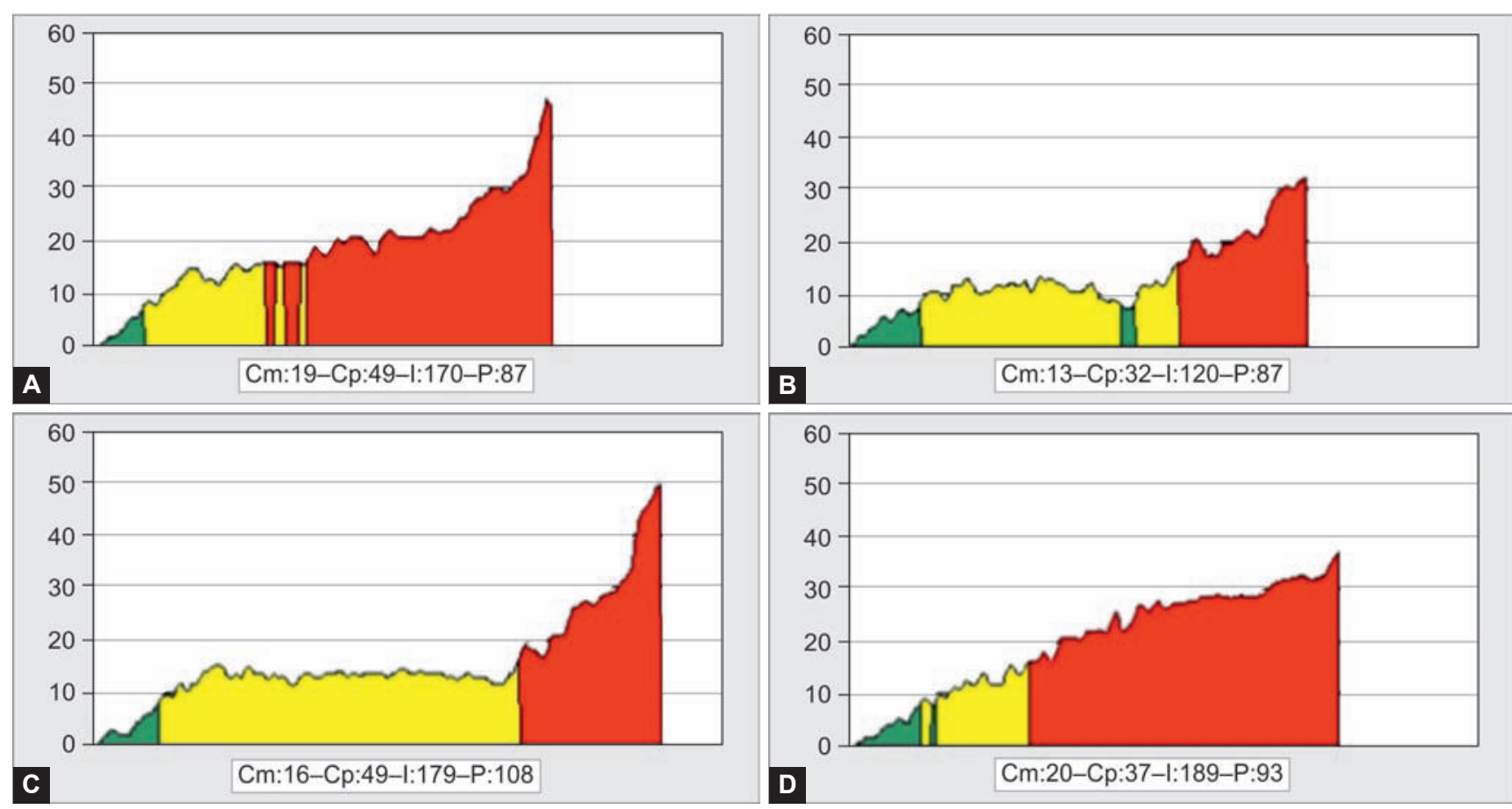

Figs 5A to D: The instantaneous torque plots recorded during implant insertion at each implant site position: 45 (A), 42 (B), 32 (C) and 35 (D). Cm: Average torque, Cp: Maximum torque, I: Integral of the torque-depth curve, P: Maximum depth reached by the probe (in tenths of millimeters)

respectively. ${ }^{26}$ Such data were interpreted as follows: site 45 was regarded as featuring an average D3 density and, in particular, a middle low-mineralized area. Position 42 was similar, with a density lower than 45 in the apical portion. At the coronal and apical portions of position 32, two D1 density zones could be observed, while the central area corresponded to D4 bone density. Finally, in position 35 , where a tilted implant was to be inserted, D1 density bone was observed in the coronal portion, becoming less dense toward the apex. The intraoperative assessment of bone density made it possible to obtain a point-topoint density measurement of the implant osteotomy, as shown in Figures $4 \mathrm{~A}$ to D. Average bone density was expressed by two parameters assessed by the device

Table 1: Bone density measured by CT scan and expressed in $\mathrm{HU}$ units

\begin{tabular}{lclll}
\hline & Position 45 & Position 42 & Position 32 & Position 35 \\
\hline Coronal & 729 & 830 & 1378 & 1160 \\
Intermediate & -18 & 30 & 190 & 130 \\
Apical & 687 & 378 & 723 & 325 \\
\hline Average & $466(\mathrm{D} 3)$ & $412.67(\mathrm{D} 3)$ & 763.67 (D3) & 538.33 (D3) \\
\hline
\end{tabular}

Table 2: Correspondence between $\mathrm{HU}$ values and Misch bone density classification according to Turkyilmaz et $\mathrm{al}^{26}$

\begin{tabular}{ll}
\hline$H U$ & Misch class \\
\hline$<150$ & D5 \\
$150-350$ & D4 \\
$350-850$ & D3 \\
$850-1250$ & D2 \\
$>1250$ & D1 \\
\hline
\end{tabular}

Table 3: Intraoperative measurements. Cm: Average torque; $\mathrm{Cp}$ : Peak torque; I: Torque-depth curve integral

\begin{tabular}{|c|c|c|c|c|}
\hline $\begin{array}{l}\text { Intraoperative } \\
\text { density } \\
\text { assessment }\end{array}$ & Position 45 & Position 42 & Position 32 & Position 35 \\
\hline $\mathrm{Cm}$ & 4 & 7 & 17 & 9 \\
\hline $\mathrm{Cp}$ & 12 & 14 & 28 & 20 \\
\hline I & 61 & 79 & 175 & 119 \\
\hline
\end{tabular}

Table 4: Measurements at implant insertion. $\mathrm{Cm}$ : Average torque; Cp: Peak torque; I: Torque-depth curve integral

\begin{tabular}{lllll}
\hline $\begin{array}{l}\text { Implant } \\
\text { insertion }\end{array}$ & Position 45 & Position 42 & Position 32 & Position 35 \\
\hline $\mathrm{Cm}$ & 16 & 13 & 19 & 20 \\
$\mathrm{Cp}$ & 49 & 32 & 49 & 37 \\
$\mathrm{I}$ & 179 & 120 & 170 & 189 \\
\hline
\end{tabular}

during probing: the average torque $(\mathrm{Cm})$ and the integral of the torque-depth function (I). These values are shown in Table 3 together with the peak torque value (Cp). Plots of the instantaneous torque-depth curves are shown for each insertion site (Figs 5A to D). The corresponding quantitative parameters $(\mathrm{Cp}, \mathrm{Cm}$ and $\mathrm{I})$ are provided in Table 4.

\section{DISCUSSION}

Bone density at implant placement sites correlates strictly with implant primary stability.$^{5-9}$ Its assessment is, therefore, of pivotal importance in planning site preparation and the loading strategy of osseointegrated implants. Computed tomography scans provide an objective and 
reliable preoperative assessment of bone density through the intensity of grey shades when measured in $\mathrm{HU}$ units. ${ }^{27}$ Such measurements correlate significantly with implant primary stability. ${ }^{28}$ Similar measurements from CBCT scans, even if dependent on the specific device being used, still correlate with histomorphometric bone density and primary implant stability. ${ }^{29-32}$ However, bone density may show ample variations even in contiguous points of the maxillary bone, and this raises the question of whether absolute or relative bone density values assessed on preoperative CTs or CBCTs will correspond to the ones at the actual placement sites, since these may not overlap perfectly with the ones preplanned on the digital scans. ${ }^{12}$ Even using stereolithographic guides for implant placement may not solve this issue since significant deviations from the preplanned placement positions may still be observed, the greatest errors being found when patients move during the CT scans, resulting in significant divergence at the level of the head and apex of the implants, and also in angular deviation. ${ }^{33-37}$ Bone density measurements with both $\mathrm{CT}$ and $\mathrm{CBCT}$ should be regarded only as indicative of the density at the actual placement site, because of topographical deviations from the planned placement position that may incur during implant positioning. Additionally, such measurements provide only preoperative information. In this sense, the traditional bone density measurement procedure based on the surgeon perception of hardness at bone drilling, corresponding to the Misch or Trisi and Rao classifications, has the advantage of being intraoperative and sitespecific. However, it is scarcely reproducible and, above all, lacks objectiveness. Data in this case report show that the device tested enabled detailed assessment of the bone density at the implant-placement site. In particular, the torque-depth plots provided detailed information about the location of areas showing greater or lower density along the osteotomy. The device allowed a finer characterization (Table 3) of the implant sites which, on the bases of the averaged CT measurements only (Table 1), appeared more homogenous as far as their density was concerned, since all could have been classified as D3 density sites. Parameters at probing and, above all, the torque-depth graph, showed a more detailed picture of the bone availability along each osteotomy. Additionally, the intraoperative assessment could be performed at an early stage of the site preparation, allowing for further adaptation of the drilling protocol according to the results of the measurement. The device also permitted measurement of a quantity at insertion, the torque-depth curve integral, correlated to the final implant BIC and the primary stability achieved, thus giving immediate feedback about the correctness of the positioning strategy. As assessing primary stability is fundamental in the choice among immediate or early or delayed loading protocols, further studies should be aimed at defining site preparation protocols and loading strategies on the basis of precise rationales, taking into account both $\mathrm{CT}$ and $\mathrm{CBCT}$ data and the bone density plots recorded by the device. ${ }^{38,39}$

\section{CONCLUSION}

The device tested provided detailed intraoperative information about the variation of bone density along the actual implant osteotomy that completed the anatomic and density data provided by CT scans.

\section{CLINICAL SIGNIFICANCE}

The device appears to be a helpful intraoperative instrument, one that facilitates adaptation of the drilling protocol and implant loading strategy to the bone density locally assessed at the implant placement site. Its routine use in a clinical setting should be assessed in controlled clinical trials involving a greater number of patients.

\section{ACKNOWLEDGMENTS}

The work was partially sponsored by IDI Evolution Srl which donated the micromotor. Data belonged to the authors and by no means did the manufacturers interfere with the conduct of the case or the publication of its results.

\section{REFERENCES}

1. Brånemark PI. Osseointegration and its experimental background. J Prosthet Dent 1983;50(3):399-410.

2. Davies JE. Mechanisms of endosseous integration. Int J Prosthodont 1998;11(5):391-401.

3. Javed F, Ahmed HB, Crespi R, Romanos GE. Role of primary stability for successful osseointegration of dental implants: Factors of influence and evaluation. Interv Med Appl Sci 2013; 5(4):162-167.

4. Chong L, Khocht A, Suzuki JB, Gaughan J. Effect of implant design on initial stability of tapered implants. J Oral Implantol 2009;35(3):130-135.

5. Friberg B, Jemt T, Lekholm U. Early failures in 4,641 consecutively placed Brånemark dental implants: a study from stage 1 surgery to the connection of completed prostheses. Int J Oral Maxillofac Implants 1991;6(2):142-146.

6. Johansson B, Back T, Hirsch JM. Cutting torque measurements in conjunction with implant placement in grafted and nongrafted maxillas as an objective evaluation of bone density: a possible method for identifying early implant failures. Clin Implant Dent Relat Res 2004;6(1):9-15.

7. Meredith N. Assessment of implant stability as a prognostic determinant. Int J Prosthodont 1998;11(5):491-501.

8. Sennerby L, Roos J. Surgical determinants of clinical success of osseointegrated oral implants: a review of the literature. Int J Prosthodont 1998;11(5):408-420. 
9. Roos J, Sennerby L, Albrektsson T. An update on the clinical documentation on currently used bone anchored endosseous oral implants. Dent Update 1997;24(5):194-200.

10. Hatcher DC, Dial C, Mayorga C. Cone beam CT for presurgical assessment of implant sites. J Calif Dent Assoc 2003;31(11): 825-833.

11. Shahlaie M, Gantes B, Schulz E, Riggs M, Crigger M. Bone density assessments of dental implant sites: 1 . Quantitative computed tomography. Int J Oral Maxillofac Implants 2003; 18(2):224-231.

12. Turkyilmaz I, Tözüm TF, Tumer C. Bone density assessments of oral implant sites using computerized tomography. J Oral Rehabil 2007;34(4):267-272.

13. De Oliveira RC, Leles CR, Normanha LM, Lindh C, RibeiroRotta RF. Assessments of trabecular bone density at implant sites on CT images. Oral Surg Oral Med Oral Pathol Oral Radiol Endod 2008;105(2):231-238.

14. Norton MR, Gamble C. Bone classification: an objective scale of bone density using the computerized tomography scan. Clin Oral Implants Res 2001;12(1):79-84.

15. Shapurian T, Damoulis PD, Reiser GM, Griffin TJ, Rand WM. Quantitative evaluation of bone density using the Hounsfield index. Int J Oral Maxillofac Implants 2006;21(2):290-297.

16. Valiyaparambil JV, Yamany I, Ortiz D, Shafer DM, Pendrys D, Freilich M, Mallya SM. Bone quality evaluation: comparison of cone beam computed tomography and subjective surgical assessment. Int J Oral Maxillofac Implants 2012;27(5): 1271-1277.

17. Mah P, Reeves TE, McDavid WD. Deriving Hounsfield units using grey levels in cone beam computed tomography. Dentomaxillofac Radiol 2010;39(6):323-335.

18. Nomura $Y$, Watanabe H, Honda E, Kurabayashi T. Reliability of voxel values from cone-beam computed tomography for dental use in evaluating bone mineral density. Clin Oral Implants Res 2010;21(5):558-562.

19. Misch, CE. Contemporary implant dentistry. St Louis: Mosby Year-Book; 1993. Density of bone: effect of treatment planning, surgical approach, and healing; p. 469-485.

20. Trisi $P$, Rao W. Bone classification: clinical-histomorphometric comparison. Clin Oral Implants Res 1999;10(1):1-7.

21. Friberg B, Sennerby L, Roos J, Johansson P, Strid CG, Lekholm U. Evaluation of bone density using cutting resistance measurements and microradiography: an in vitro study in pig ribs. Clin Oral Implants Res 1995;6(3):164-171.

22. Friberg B, Sennerby L, Roos J, Lekholm U. Identification of bone quality in conjunction with insertion of titanium implants. A pilot study in jaw autopsy specimens. Clin Oral Implants Res 1995;6(4):213-219.

23. Iezzi G, Scarano A, Di Stefano D, Arosio P, Doi K, Ricci L, Piattelli A, Perrotti V. Correlation between the bone density recorded by a computerized implant motor and by a histomorphometric analysis: A preliminary in vitro study on bovine ribs. Clin Implant Dent Relat Res 2015;17 suppl 1:e35-44.

24. Di Stefano DA, Arosio P, Pagnutti S. A possible novel objective intraoperative measurement of maxillary bone density. Minerva Stomatol 2013;62(7-8):259-265.

25. Capparé P, Vinci R, Di Stefano DA, Traini T, Gherlone EF, Gastaldi G. Correlation between initial BIC and the insertion torque-depth Integral recorded with an instantaneous torque- measuring implant motor: an in vivo study. Clin Implant Dent Relat Res 2015 [in press].

26. Turkyilmaz I, McGlumphy EA. Influence of bone density on implant stability parameters and implant success: a retrospective clinical study. BMC Oral Health 2008;8:32.

27. Akdeniz BG, Oksan T, Kovanlikaya I, Genç I. Evaluation of bone height and bone density by computed tomography and panoramic radiography for implant recipient sites. J Oral Implantol 2000;26(2):114-119.

28. Pagliani L, Motroni A, Nappo A, Sennerby L. Short communication: use of a diagnostic software to predict bone density and implant stability in preoperative CTs. Clin Implant Dent Relat Res 2012;14(4):553-557.

29. González-García R, Monje F. The reliability of cone-beam computed tomography to assess bone density at dental implant recipient sites: a histomorphometric analysis by micro-CT. Clin Oral Implants Res 2013;24(8):871-879.

30. Monje A, Monje F, González-García R, Galindo-Moreno P, Rodriguez-Salvanes F, Wang HL. Comparison between microcomputed tomography and cone-beam computed tomography radiologic bone to assess atrophic posterior maxilla density and microarchitecture. Clin Oral Implants Res 2014;25(6):723-728.

31. Marquezan M, Osório A, Sant'Anna E, Souza MM, Maia L. Does bone mineral density influence the primary stability of dental implants? A systematic review. Clin Oral Implants Res 2012;23(7):767-774.

32. Isoda K, Ayukawa Y, Tsukiyama Y, Sogo M, Matsushita Y, Koyano K. Relationship between the bone density estimated by cone-beam computed tomography and the primary stability of dental implants. Clin Oral Implants Res 2012;23(7):832-836.

33. D'haese J, Van De Velde T, Komiyama A, Hultin M, De Bruyn H. Accuracy and complications using computer-designed stereolithographic surgical guides for oral rehabilitation by means of dental implants: a review of the literature. Clin Implant Dent Relat Res 2012;14(3):321-335.

34. Pettersson A, Komiyama A, Hultin M, Näsström K, Klinge B. Accuracy of virtually planned and template guided implant surgery on edentate patients. Clin Implant Dent Relat Res 2012;14(4):527-537.

35. Cassetta M, Stefanelli LV, Giansanti M, Calasso S. Accuracy of implant placement with a stereolithographic surgical template. Int J Oral Maxillofac Implants 2012;27(3):655-663.

36. Pettersson A, Kero T, Gillot L, Cannas B, Fäldt J, Söderberg R, Näsström K. Accuracy of CAD/CAM-guided surgical template implant surgery on human cadavers: Part I. J Prosthet Dent 2010;103(6):334-342.

37. Beretta M, Poli PP, Maiorana C. Accuracy of computer-aided template-guided oral implant placement: a prospective clinical study. J Periodontal Implant Sci 2014;44(4):184-193.

38. Prosper L, Crespi R, Valenti E, Cappare P, Gherlone E. Fiveyear follow-up of wide-diameter implants placed in fresh molar extraction sockets in the mandible: immediate versus delayed loading. Int J Oral Maxillofac Implants 2010;25(3): 607-612.

39. Crespi R, Capparé P, Gherlone E, Romanos GE. Immediate versus delayed loading of dental implants placed in fresh extraction sockets in the maxillary esthetic zone: a clinical comparative study. Int J Oral Maxillofac Implants 2008; 23(4):753-758. 